\title{
EXTREMAL LENGTH AND REMOVABLE BOUNDARIES OF RIEMANN SURFACES
}

\author{
BY BURTON RODIN ${ }^{1}$ \\ Communicated by Maurice Heins, September 30, 1965
}

1. Introduction. Given a Riemann surface $R$ let $K D$ denote the space of harmonic functions $u$ on $R$ with finite Dirichlet norm $\|d u\|$ and such that $* d u$ is semiexact, i.e., $\int_{c} * d u=0$ for all dividing cycles $c$. Then $O_{K D}$ denotes the class of Riemann surfaces $R$ for which every function in $K D$ is constant. Clearly $O_{H D} \subset O_{K D} \subset O_{A D}$ and for planar surfaces $O_{K D}=O_{A D}$. Under various names, this class $O_{K D}$ has been studied by many authors (see, for example, Royden [4], Sario [5]).

The concept of the extremal length $\lambda(\mathcal{F})$ of a family $\mathscr{F}$ of curves on a Riemann surface $R$ can be extended to the case that $\mathscr{F}$ is a family of curves on the Kerékjártó-Stoilöw compactification $\hat{R}$ of $R$ merely by eliminating the ideal points from each curve. Let $\alpha_{0}, \alpha_{1}$ be compact subsets of $R$. Define $\hat{F}$ to be the family of all arcs on $\hat{R}$ with initial point in $\alpha_{0}$ and endpoint in $\alpha_{1}$. Define $\mathcal{F}$ to be the subfamily of $\hat{F}$ consisting of all arcs in $R$. We consider two notions for the extremal distance between $\alpha_{0}$ and $\alpha_{1}$, viz., define

$$
\lambda\left(\alpha_{0}, \alpha_{1}\right)=\lambda(\mathfrak{F}), \quad \hat{\lambda}\left(\alpha_{0}, \alpha_{1}\right)=\lambda(\hat{F}) .
$$

The aim of this note is to announce the following

THeOREM. A necessary and sufficient condition that $\lambda\left(\alpha_{0}, \alpha_{1}\right)=\hat{\lambda}\left(\alpha_{0}, \alpha_{1}\right)$ for all compact subsets $\alpha_{0}, \alpha_{1}$ of $R$ is that $R \in O_{K D}$.

Our Theorem is reminiscent of the already classical result of Ahlfors-Beurling [1]:

$A$ plane point set $E$ is an $A D$-null set if and only if the removal of $E$ does not change extremal distances.

The relationship between these results will be discussed in $\$ 3$ below.

2. Sketch of the proof. The complete proof will appear in a forthcoming book [3]. The main steps in proving the necessity of the extremal distance condition are the following. (i) To construct functions $u, \hat{u}$ on $R$ such that $\lambda\left(\alpha_{0}, \alpha_{1}\right)=\|d u\|^{-2}$ and $\hat{\lambda}\left(\alpha_{0}, \alpha_{1}\right)=\|d \hat{u}\| \|^{-2}$, (ii) to show that $R \in O_{K D}$ implies $u=a$. (Actually, these steps are applied to each component of $R-\alpha_{0}-\alpha_{1}$, rather than $R$ itself.)

1 This work was supported in part by the National Science Foundation under grant GP 4106 at the University of California, San Diego. 
Step (i) was accomplished in [2]. There it is shown that if $\alpha_{0}, \alpha_{1}$, $\gamma_{0}, \gamma_{1}$ is an (admissible) partition of the ideal boundary of a Riemann surface $S$ then a harmonic function $u\left(\alpha_{0}, \alpha_{1}, \gamma_{0}, \gamma_{1}\right)$ on $S$ can be constructed which is determined by the following conditions-they are to be interpreted in the sense of a limit via an exhaustion of $S$ : (1) $u\left(\alpha_{0}, \alpha_{1}, \gamma_{0}, \gamma_{1}\right) \equiv 0$ on $\alpha_{0}$, (2) $u\left(\alpha_{0}, \alpha_{1}, \gamma_{0}, \gamma_{1}\right) \equiv 1$ on $\alpha_{1}$, (3) $u\left(\alpha_{0}, \alpha_{1}, \gamma_{0}, \gamma_{1}\right)$ has vanishing normal derivative along $\gamma_{0}\left(L_{0}\right.$-behavior near $\left.\gamma_{0}\right)$, (4) along each component of $\gamma_{1}, u\left(\alpha_{0}, \alpha_{1}, \gamma_{0}, \gamma_{1}\right)$ is constant and has vanishing flux ( $L_{1}$-behavior near $\left.\gamma_{1}\right)$. Furthermore, it is shown that $\left\|d u\left(\alpha_{0}, \alpha_{1}, \gamma_{0}, \gamma_{1}\right)\right\|^{-2}=\lambda\left(\mathcal{F}\left(\alpha_{0}, \alpha_{1}, \gamma_{0}, \gamma_{1}\right)\right)$ where $\mathcal{F}\left(\alpha_{0}, \alpha_{1}, \gamma_{0}, \gamma_{1}\right)$ is the family of arcs on $S \cup \alpha_{0} \cup \alpha_{1} \cup \gamma_{1}$ with initial point in $\alpha_{0}$ and endpoint in $\alpha_{1}$. Step (i) now follows since we have $u=u\left(\alpha_{0}, \alpha_{1}, \beta, \phi\right)$ and $\hat{u}=u\left(\alpha_{0}, \alpha_{1}, \phi, \beta\right)$ where $\beta$ is the ideal boundary of $R$.

Step (ii) is accomplished by showing that on any $S \in O_{K D}, L_{0^{-}}$and $L_{1}$-behavior cannot be distinguished.

To prove that the extremal distance condition of the Theorem is sufficient for $R \in O_{K D}$ we consider a consequence of the assumption $u=\hat{u}$ when $\alpha_{0}, \alpha_{1}$ vary over two systems of concentric disks centered at points $\zeta_{0}, \zeta_{1}$ of $R$. For $i=1,2$ let $p_{i}$ denote a harmonic function on $R$ with simple logarithmic poles at $\zeta_{0}$ and $\zeta_{1}$ of opposite sign, and with $L_{i}$-behavior near the ideal boundary $\beta$ of $R$. As a limiting case of $u=\hat{u}$ we derive $p_{0}=p_{1}+$ constant. In general, the differential $\psi=d p_{1}-d p_{0}$ has the reproducing property

$$
\iint_{R} d h \wedge * \psi=2 \pi \int_{\zeta_{0}}^{\zeta_{1}} d h
$$

for all $h \in K D$. Since $\psi=0$ in our case, $R \in O_{K D}$.

3. Remarks. Let $R$ be a region in the extended plane $\boldsymbol{P}$ and let $E=P-R$. Then $\lambda\left(\alpha_{0}, \alpha_{1}\right)$ is the usual extremal distance between $\alpha_{0}, \alpha_{1}$ on $P-E$. Let $\lambda_{P}\left(\alpha_{0}, \alpha_{1}\right)$ denote the extremal distance between $\alpha_{0}, \alpha_{1}$ on $P$. Then we have

$$
\lambda\left(\alpha_{0}, \alpha_{1}\right) \geqq \lambda_{P}\left(\alpha_{0}, \alpha_{1}\right) \geqq \hat{\lambda}\left(\alpha_{0}, \alpha_{1}\right) .
$$

Thus our Theorem immediately implies the "only if" part of the Ahlfors-Beurling Theorem.

To derive the converse, assume that the removal of $E$ does not change extremal distances. As in $\$ 2$ we see that this implies that $E$ is a removable singularity for the function $p_{0}$ defined on $R$. In general, the partial derivative of $p_{0}$ with respect to $\operatorname{Re} \zeta_{1}$ yields the real part of the horizontal slit mapping function for $R$ with pole at $\zeta_{1}$. It 
follows that $E$ is a removable singularity for any parallel slit mapping. Thus the span of $R$ vanishes; hence $R \in O_{A D}$.

\section{REFERENCES}

1. L. V. Ahlfors and A. Beurling, Conformal invariants and function-theoretic null sets, Acta Math. 83 (1950), 101-129.

2. A. Marden and B. Rodin, Extremal and conjugate extremal distance on open Riemann surfaces with applications to circular-radial slit mappings. Acta Math. 115 (1966).

3. B. Rodin and L. Sario, Principal functions, Van Nostrand, Princeton, N. J. (to appear).

4. H. L. Royden, On a class of null-bounded Riemann surfaces, Comment. Math. Helv. 34 (1960), 52-66.

5. L. Sario, An extremal method on arbitrary Riemann surfaces, Trans. Amer. Math. Soc. 73 (1952), 459-470.

University of California, San Diego 